Trends in research and development for future detectors

This content has been downloaded from IOPscience. Please scroll down to see the full text. 2013 Phys. Scr. 2013014017

(http://iopscience.iop.org/1402-4896/2013/T158/014017)

View the table of contents for this issue, or go to the journal homepage for more

Download details:

IP Address: 137.138.125.164

This content was downloaded on 20/05/2014 at 08:44

Please note that terms and conditions apply. 


\title{
Trends in research and development for future detectors
}

\author{
Ariella Cattai \\ CERN, Department of Physics, CH-1211 Geneva 23, Switzerland \\ E-mail: Ariella.Cattai@cern.ch
}

Received 1 October 2013

Accepted for publication 7 November 2013

Published 6 December 2013

Online at stacks.iop.org/PhysScr/T158/014017

\begin{abstract}
Development of novel detector concepts has always played a major role in supporting and enabling scientific research. In the forthcoming phase of high energy physics (HEP), the design and development of new detectors and detector concepts will be even more important than it was in the past owing to the harsh environmental conditions and the challenging requests imposed by the physicists' needs for: improved spatial and time resolution, innovative functions, acquisition speed, radiation tolerance, minimal power consumption, robustness and reliability, minimal material and more. This overview addresses the challenges that upgrades and future projects in HEP will impose in terms of novel technologies and stresses the detectors' potential and limitations in attempting to achieve the scientific goals. In addition the increasingly strong dependence on large-scale industrial production and industrial development, especially in the area of integrated electronics, sensors and large complex systems will be addressed.
\end{abstract}

PACS number: 07.77.Ka

(Some figures may appear in colour only in the online journal)

\section{Preface}

When reading the new proposals for the upgrade of existing experiments or for the new ones in pioneering machines, the attentive expert will rarely find any striking novelties. This is a reality: the well-proven detector technologies in use in experiments for many years, are still the major protagonists on the scene: time projection chamber (TPC), resistive plate chamber (RPC), ring-imaging Cherenkov detector (RICHs) and all other friends, are still there, ancient-but yet modern-soldiers in the battlefield of research ${ }^{1}$. Ancient is the correct word! wire chambers by Charpak et al [1] in 1968, TPC by Nygren and Marx [2, 3] in 1974, RICHs by Seguinot and Ypsilantis [4] in 1977, RPC by Santonico and Cardarelli [5] 1981, micro pattern gaseous detector (MPGD) by Giomataris and Sauli in the late 1990s [6-8] and three-dimensional (3D) silicon pixels by Parker et al [9] in 1997, none of these glorious detectors was developed for or within an experiment: they all sprouted in the Palaeozoic era when research and development (R\&D) was a natural part of

1 TPC: time projection chamber; RICH: ring-imaging Cherenkov detector; RPC: resistive plate chamber; MPGD: micro pattern gaseous detector. research. Why this trend no longer persist? Today, R\&D on detectors is increasingly driven by the necessities of ongoing or upcoming experiments ${ }^{2}$, therefore it is carried out within well-defined boundary conditions and limiting constraints. Is this an effect attributable to the limited funding? Or to the organization of the high energy physics (HEP) community, perhaps? It is difficult to give a definitive response, but this is a very interesting phenomenon and it should not be underestimated. In fact, if the present trend continues, it will be almost impossible to exploit revolutionary detection systems in future experiments, with a real substantial risk of obstructing emerging technologies.

Future experiments will require complex detectors in which high precision elements are integrated into innovative large-scale apparatuses with a strong interdependence between the detection technologies, mechanics, electronics and computing. A few questions stand out: will the world of detectors move increasingly towards industrial production, where, to keep costs contained and to ease serial production,

\footnotetext{
2 A survey launched by ICFA and ECFA in 2012 among physicists in the European Community confirmed that $85 \%$ of R\&D for detectors is done within large experiments.
} 

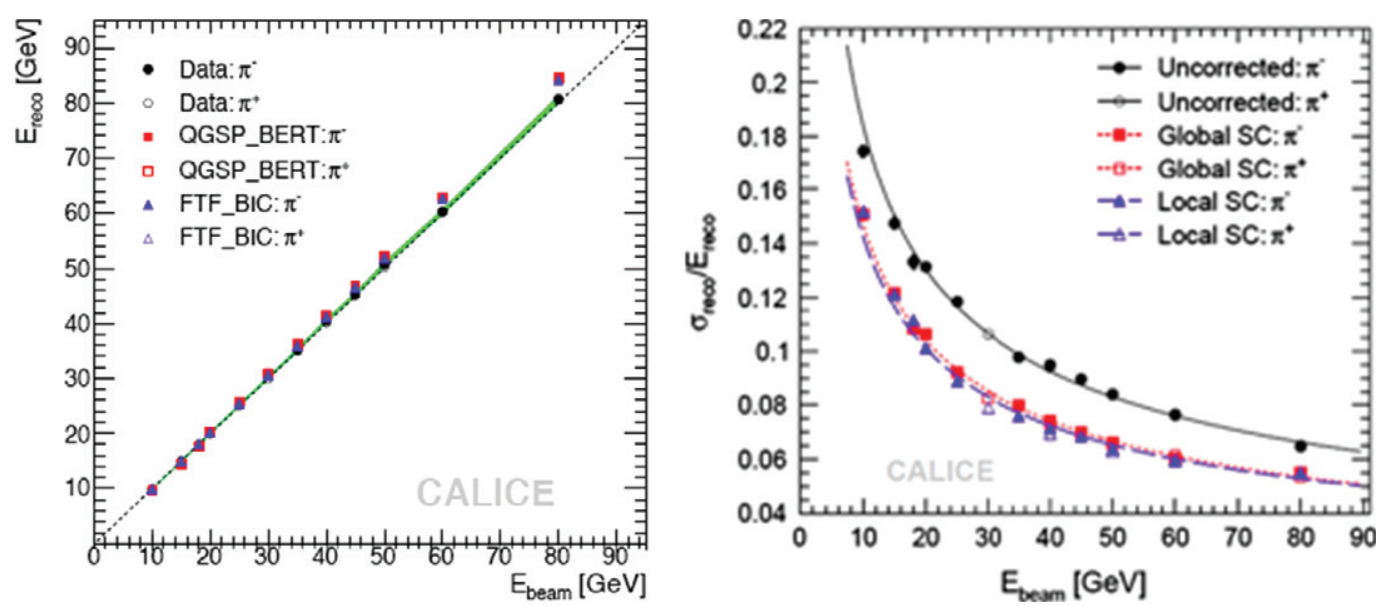

Figure 1. (Left) Linear response of an analog hadron calorimeter, made of steel-scintillator tiles, versus beam energy. Circles refer to data from pions, squares and triangles refer to two simulations. (Right) Energy resolution of an analog hadron calorimeter. The black circles are the uncorrected data, the squares and triangles are the data corrected with the software compensation techniques made possible with the unprecedented segmentation of imaging calorimeters [17].

some of the technical specifications are relaxed? As we come to rely more and more on micro-technologies and on industry, will we be able to cope with R\&D costs set by other commercial players? Can we afford several fault-tolerant designs and process technologies and will this be cost effective? Can the high yield that we request be achieved at low production cost? Despite our limited commercial impact for industry, can we still hope to be among the major players? Will we ever be able to come back to the ingenious artisanal manufacture that has always been the distinctive high-quality product of our community? Or will we have lost the know-how so that we would not even be capable of delivering at the prototyping level?

Several of the researchers at the major detectors are facing these questions and in the following sections this topic will be approached in the context of the present status of the technologies and the expected evolution.

\section{Calorimetry: from art to series production}

Is the slowdown of $R \& D$ in calorimetry the first example of the phenomenon just described?

It is incontrovertible that calorimetry has long been a linear collider driven effort [10-12], where the need to separate heavy bosons, $W$ and $Z$, by reconstructing their invariant masses in di-jet final states on an event-by-event basis, imposes significant improvements in jet energy resolution with respect to today's systems. Being the target: $\sigma_{\text {jet }}=30 \% / \sqrt{E_{\text {jet }}}$. To achieve such a goal the future experiments focus on the particle flow algorithm (PFA): a method that aims to reconstruct each particle in a jet individually and to optimize the jet energy resolution by combining tracking and calorimetric measurements subsequent to a judicious assignment of energy deposited [13-15]. Where energy reconstructed in the calorimeters belongs to an incident charged particle, it will be ignored and replaced by the corresponding momentum measurement, however, if it was produced by neutral particles, it will be kept in the counting. The choice of being capable of correctly associating energy deposits to charged or neutral particles imposes a number of basic and unavoidable requirements on the calorimeter systems, the most stringent one being the necessity for the electromagnetic and hadronic sections to have imaging capabilities that allow both efficient track-following and correct assignment of energy clusters to tracks. These requirements imply that the calorimeters must be subtly segmented both longitudinally and transversely. Furthermore, the calorimeters must be located inside the magnetic coil with minimal dead space and, to maintain a good energy resolution, the sampling thickness must be carefully optimized.

In recent years, we have assisted in an enormous effort carried on by the community to bring PFA-based calorimeters from small prototypes to the status of well-established technologies. Several possibilities were considered for the active detectors, all of them crucially relying on highly integrated low power electronics: gas electron multipliers (GEMs), MicroMegas, RPCs, silicon and scintillators. Accurate test beam campaigns performed on very large installations over many years have confirmed the feasibility of imaging calorimeters and demonstrated their excellent performances [16].

As an example figure 1 (left) shows the beam energy versus the reconstructed energy in an analogue hadron calorimeter with plastic tiles as the active media, read-out by silicon photomultipliers (SiPM). The high granularity of imaging calorimeters can be advantageously exploited to correct, on an event-by-event basis, the fluctuations of the electromagnetic energy fraction in the hadronic showers. As shown in figure 1 (right), by applying the software compensation methods, the energy resolution can be improved by some $20 \%$, thereby achieving a good hadron energy resolution [17] of the order of $45 \% / \sqrt{E}$.

But there is a price to pay for imaging calorimetry: depending on the technologies, the active areas of the future calorimeters are in the range 1200-2500 and 4000-7000 $\mathrm{m}^{2}$ for the electromagnetic and hadronic sections respectively; consequently the electronic channels arrive at the diverging 
number of $10^{7}-10^{8}$ units, implying a need for a challenging cell-by-cell calibration. Hence, after the initial phase that proved the working principle of PFA imaging calorimetry via experimental data and simulation, the community is entering a phase where compromises between the technological feasibility and the optimization of performance have to be evaluated and straightened out. Issues of integration, scalability and complex large systems, have to be addressed and solved within financial boundaries, distracting the attention of physicists, probably for many years, from fundamental research in favour of industrialization and serial production. The chances of jeopardizing innovative discoveries in the calorimetry realm, including restraining progresses in homogeneous techniques and scintillating crystals fields so important for civil applications, is perceptible.

\section{Tracking and electronics: the polarizing issues}

Microelectronics technologies have been key to the success of tracking systems and have paved the road to other technologies that are appropriate for building detectors (see section 5) of promising potential and performances. Future HEP experiments will need to have, among other things, better vertex reconstruction, very fine granularity, complex trigger capabilities and high data exchange rates, a low material budget, higher radiation hardness, and more. These requests have a direct impact on the specifications of the front-end and on-detector electronics. Today, very deep-submicron technologies could meet the future experimental requirements while the emerging interconnect technologies, like wafer to wafer bonding, through-silicon-vias (TSV) and 3D vertical assembly, could provide viable solutions to the compelling requests of achieving high-density trackers suited to efficiently reconstructed physics events at high luminosity and high pile-up, or in the presence of very high backgrounds [18].

\subsection{Granularity and interconnections}

Efficient primary and secondary vertex reconstructions of unprecedented accuracy are crucial for many physics analyses; hence, vertex detectors realized with pixels in monolithic or hybrid technologies, have become key elements in HEP experiments. In the future, their role will still be central and the requirements imposed on the detectors will be even more stringent; for example, granularity as low as $25 \mu \mathrm{m}$ or less and minimal material budget of the order of $0.1 \% \mathrm{X}_{0}$ per layer. The necessity of reducing the front-end pitch has several repercussions: the hybrid pixel technology, that has so far monopolized the field and has proven to be a mature and successful choice in the present experiments, will need to stand up to vigorous $\mathrm{R} \& \mathrm{D}$ on interconnections if it is to maintain its prominent role; the total amount of functionalities that can be included in the front-end electronics will be limited by the reduced size of the read-out cell; forcing high-speed active devices into narrow and crowded elementary areas might increase the power consumption and dissipation per unit of volume. Various approaches are being

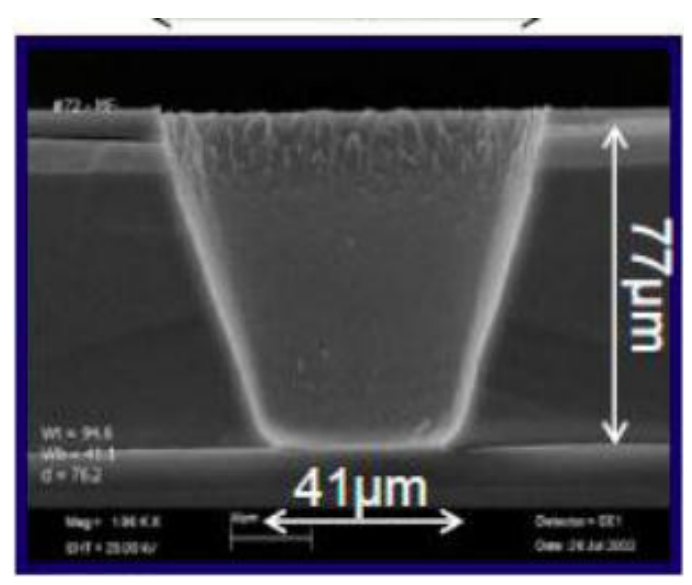

Figure 2. Detail of a single tapered TSV processed by Fraunhofer IZM. The micro-metric precision of this technology is highly remarkable. (Courtesy: University of Bonn/IZM, Berlin).

pursued to tackle these challenges, amongst which the most advanced ones:

- Several forms of 3D integrated assembly to interconnect multiple layers of semiconductor wafers of different functionalities with the aim of obtaining more compact designs for the modules [19, 20].

- Advanced interconnections to replace the traditional wire or bump bonding [21]. In this field, many efforts are invested on two very promising techniques: the TSV [22] to interconnect layers and to drive the chip contacts to the back side of the ASIC instead of routing them to the wire bonding pads located on the edges of the chip (see figure 2); the solid-liquid inter-diffusion technique (SLID) [23] characterized by a very thin eutectic $\mathrm{Cu}-\mathrm{Sn}$ alloy that joins the sensor and the readout chip contact pads. Due to the low temperatures exploited during the manufacture of the SLID connections, this process is extraordinarily well-suited for the production of multi-layer assemblies. Although the efficiency of these modules depends dramatically on the SLID-pad sizes, pitches, aplanarities and on the alignment between sensor and read-out chip, prototype modules demonstrated that full connectivity efficiency can also be achieved for irradiated modules and after severe thermal cycling. Hence, this development paves the road to hybrid pixel modules, for the future detectors, with contact pads of different shapes and pitch size as low as $20 \mu \mathrm{m}$, sensor and readout chips down in the 75-150 and $60 \mu \mathrm{m}$, respectively [24, 25].

Monolithic pixels, in which the thin pixel elements are integrated with the readout electronics offer another natural and powerful option to achieve small pitch sizes. These detectors can combine high speed and complex signal processing in each pixel; they offer lower capacity and low power consumption $\left(P_{\text {avg }}<50 \mathrm{~mW} \mathrm{~cm}^{-2}\right)$ when powered in pulsing mode and can be realized within a material budget well below the LHC standards. Relatively slow monolithic detectors, DEPFET and MAPS, with a row by row read-out, are going to be implemented soon for the upgrade of the BELLE and STAR experiments, respectively [26]. MAPS are also the preferred option for attaining the aggressive 

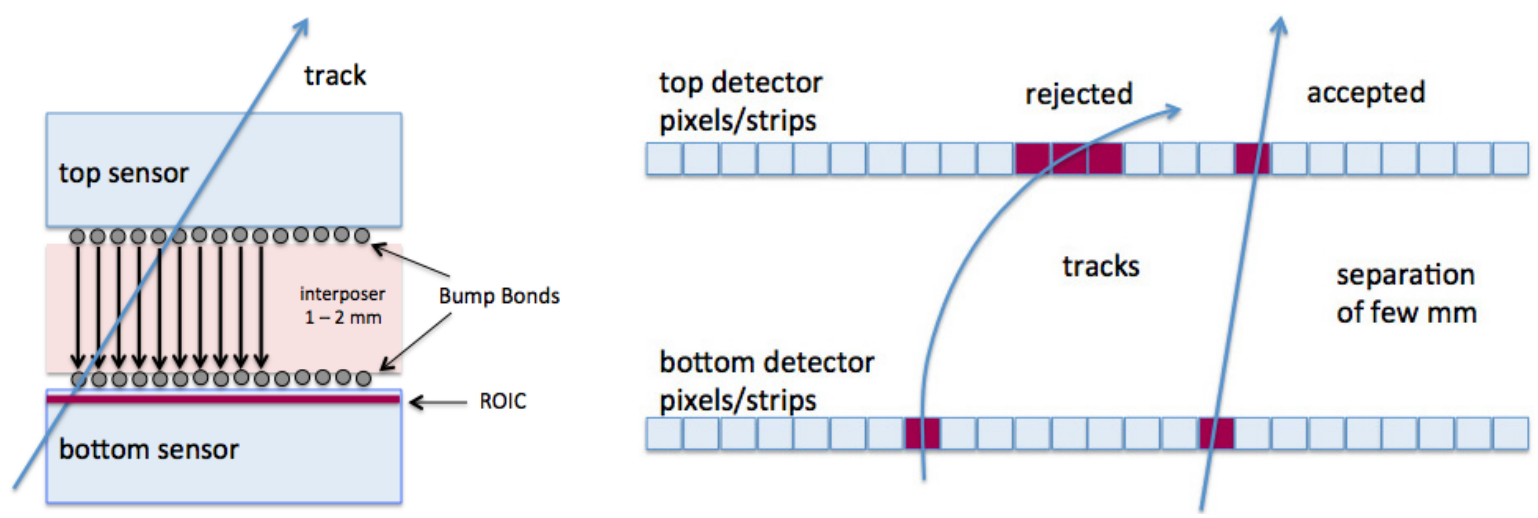

Figure 3. (Left) Concept of a pair of top-bottom sensors assembled with a small radial distance and communicating with each other through an interposer; (right) small track segments can be identified by a coincidence between the top and bottom pixel sensors. The process information can be used at the trigger level.

target of $0.3 \%$ of radiation length per layer in the ALICE inner tracking system upgrade. Very elegant developments are being pursued for applications relevant to future linear colliders: the Chronopixel [27] and the hybrid CLICpix [28] concepts. They are currently realized in dense (90 and $65 \mathrm{~nm}$ ) very deep sub-micron technologies, promising solutions for the integration of high density circuitry and high speed functionalities in small elementary cells of $25 \mu \mathrm{m} \times$ $25 \mu \mathrm{m}$. These developments also face another extraordinary challenge: time-tagging the hits in an event with single bunch precision to significantly reduce the background. In CLIC, to separate beam-induced background from physics events, time slicing with an accuracy of $10 \mathrm{~ns}$ will be required.

Although monolithic pixels offer many advantages, one should notice that the manufacturing relies on industrial procedures optimized for commercial purposes, which may differ substantially from the needs of particle detection and may induce some limitation in the performances.

\subsection{Advanced trigger logic}

Another way to cope with the high hit rate, with up to hundreds of pile-up events per bunch-crossing @ $5 \times$ $10^{34} \mathrm{~cm}^{-2} \mathrm{~s}^{-1}$ in HL-LHC, is to adopt a more sophisticated trigger logic. Track elements, or vertices, could be constructed with the single hits by adopting prompt on-detector processing; hardware-based pattern recognition could then be exploited to improve the level-2, or level-1, trigger and reject low $p_{\mathrm{T}}$ tracks. These complex processing and advanced readout architectures can be very elegantly implemented exploiting the 3D vertical integration (3D-VI) technologies, just described. A 3D chip is formed of several thin layers of semiconductor, interconnected to form a compact circuit; each layer is optimized for a specific task thereby improving speed, reducing cross-talk and increasing the functionality. A generic compact detector 3D-VI suitable to implement advanced trigger logic is pictured in figure 3 on the left: two layers of pixel sensors are placed close together with small (few $\mathrm{mm}$ ) radial separation to house passive interposers and layers of active electronics. Raw signals from particles impinging in the top and bottom sensors are transported to the middle electronic layers by means of conductive vias; small track segments can be identified with coincidence hits from the top-bottom layers (figure 3 right) and the processed information is then passed to the trigger to accept or reject tracks corresponding to a certain momentum range [29-32].

A detailed simulation, performed in the framework of the CMS HL-LHC upgrade demonstrates that implementing this trigger logic, even on only one vertex layer of the tracker system, would reduce the detector data rate by a factor of 20 , while maintaining a track-finding efficiency in excess of $96 \%$ for tracks with $p_{\mathrm{T}}>2 \mathrm{GeV} c$ [33].

Obviously, there are a number of challenges in the elegant approach of novel 3D-VI detectors: the complexity of the interconnections between different layers required; the capability of mastering the fabrication of electrically isolated connections through the silicon substrate; the ability to thin the substrate below $50 \mu \mathrm{m}$ and the need for very high precision on the inter-layer alignment. At present the 3D development is driven by high volume commercial applications, hence, the access to these new technologies requires substantial financial resources and there may be a mismatch between requirements from industry and HEP. To overcome the last problems, it is necessary to sustain and foster the network between R\&D groups working in this field, notably research institutes, industry and foundries [20] and to encourage shared engineering and production runs between different projects.

\subsection{Radiation hardness}

Extreme conditions await the vertex detectors positioned at very low radius in HL-LHC experiments. Fluences up to $10^{16} \mathrm{n}_{\mathrm{eq}} \mathrm{cm}^{-2}$ after $3000 \mathrm{ft}^{-1}$ and the ionizing dose of $10 \mathrm{MGy}$ will degrade the thick sensors now in use in the experiments, dramatically affecting most of the relevant physical parameters, perturbing the space charge and the electrical field across the sensors, inducing charge trapping and reducing the signals' amplitude, increasing the leakage current, noise and power dissipation and ultimately limiting the charge collection efficiency (CCE) of the detectors. The radiation hardness problem is obviously one that is not underestimated by the community, and nowadays the $R \& D$ on sensors is targeted towards the production of a new generation of silicon detectors where the geometry of the sensors has been comprehensively simulated and tested to ensure that very high fields can be applied across the sensitive area; the most successful achievements in the field are the thin, planar 

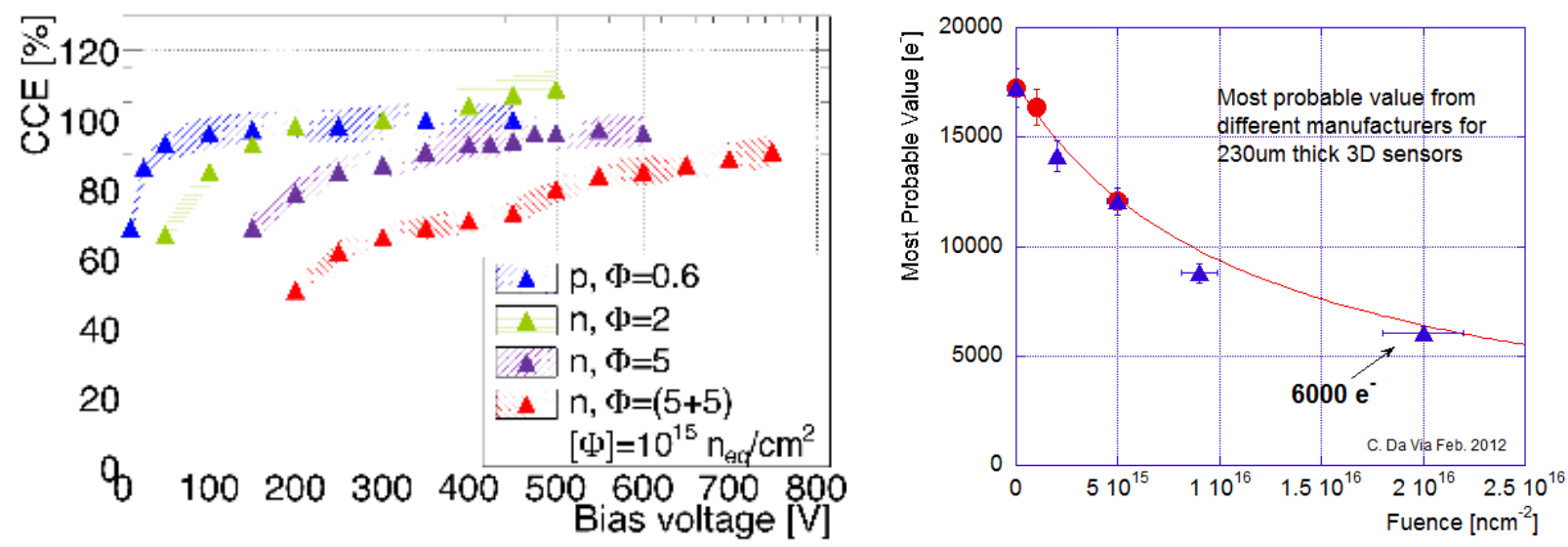

Figure 4. (Left) CCE, normalized to the charge before irradiation, as a function of the bias voltage, for different fluences obtained with the ATLAS $75 \mu \mathrm{m}$ thin n-in-p sensors interconnected to the read-out chips by means of SLID technology (Courtesy of A Macchiolo). (Right) Signal response as a function of fluences for ATLAS 3D-Silicon pixels from three different producers. At $10^{16} \mathrm{n}_{\mathrm{eq}} \mathrm{cm}^{-2}$ and $200 \mathrm{~V}$ bias, $\sim 40 \%$ of the initial signal corresponding to 6000 electrons is detected (Courtesy of C Da Via).

detectors [25, 34] and the 3D sensors [35-37], both in hybrid technology.

Recent results (figure 4) show that both of these types of hybrid detectors may reach the level of radiation hardness necessary to equip the first layers of the experiments at HL-LHC, provided that the observed charge multiplication effects [38] at higher voltages can be controlled and made uniform across the detection area. All the innumerable detectors in monolithic technology have, however, been found to have radiation hardness ranging from a few $10^{12} \mathrm{n}_{\mathrm{eq}} \mathrm{cm}^{-2}$ to $10^{14} \mathrm{n}_{\mathrm{eq}} \mathrm{cm}^{-2}$, hence this makes them most suitable for operating at the lepton colliders. One may now recall that an indispensable requirement for the vertexes is also the spatial resolution, a feature certainly guaranteed by the monolithic pixels but not yet, on a large scale, by the hybrids. Here we face a paradox: hybrids show radiation hardness, but limited granularity, monolithic detectors have the opposite properties; this is a compelling reason for pursuing a methodical R\&D campaign on radiation hardness, sustained by simulation studies, for both hybrid and monolithic technologies and for undertaking systematic research on advanced inter-connections.

\subsection{Prototyping, readiness level and connection with industry}

From the moment in which a new technology is first conceptualized, it takes many refinements and increasingly realistic testing before it can be considered to have been sufficiently well proven for it to be incorporated into an experiment. The technology readiness level (TRL) is a measure used to assess the maturity of evolving technologies from the initial conceptual stage to the early operations.

Figure 5 shows the evolution of the readiness level of a few well established pixels technologies in use in existing experiments; the time lapse from the conceptual phase to the implementation in an experiment is substantial, ranging from 10 to 20 years. The implications of such long intervals should be analysed in conjunction with the progresses of the semiconductor industry. Over the past 25 years, the minimum lithographic feature size in CMOS technology made it possible for a doubling of the transistor densities

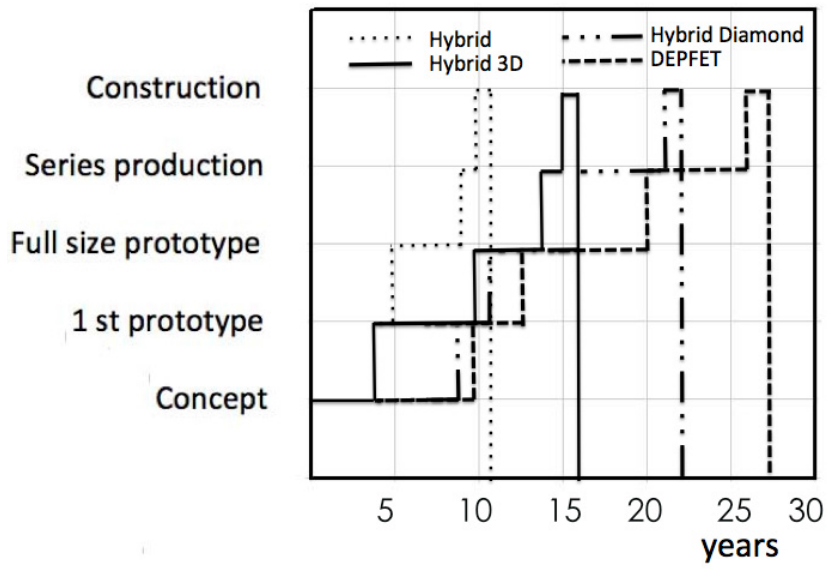

Figure 5. TRL of major pixel detector technologies in use. The time elapsed from the original conception to the implementation in an experiments is a function of the complexity of the technology and can arrive up to more than 20 years.

to take place roughly every two years, thereby attaining the 16 and $22 \mathrm{~nm}$ regime of today's applications (figure $6 \mathrm{left}$ ), while the HEP world unfortunately remained well behind. For such advanced technologies, the design and mask costs continue to rise, arriving at a present level of millions of dollars for $22 \mathrm{~nm}$ complex CMOS processes (figure 6 right).

Very deep-submicron technologies open totally new possibilities for building detectors with increasing capabilities and performance, but their complexity and cost, as well as their qualification for our harsh environment, will require a very substantial investment in terms of both money and manpower. While industry has invested in equipment and human resources at an exponentially increasing rate, HEP has barely been able to maintain the same level of investment over the years in a field that evolves very rapidly; hence, to successfully face the upcoming challenges, our community needs to select and pursue $R \& D$ on alternative technological options in parallel, needs to choose among the technological options expected to be available longer in time and needs to co-ordinate all efforts to provide common and organized technical and financial support. 

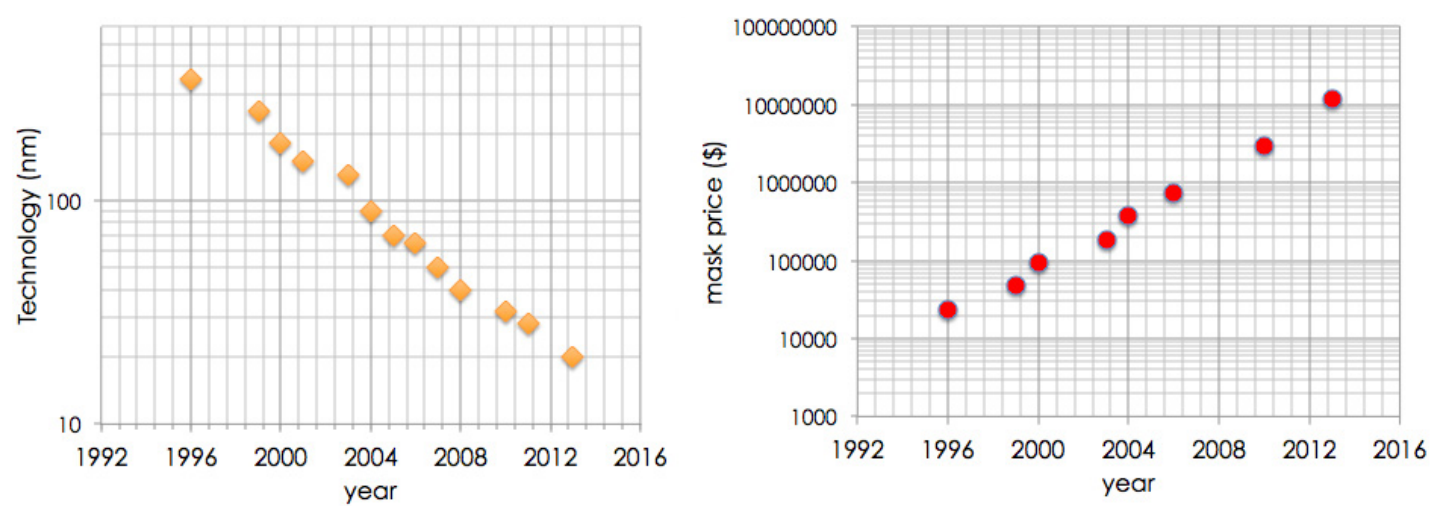

Figure 6. Rough evolution along the year of: (left) minimum lithographic feature size in CMOS technology; (right) average cost per lithographic mask. Note that several masks are required for the realization of a chip, depending on its complexity, hence the financial investment is considerable $[39,40]$.

\section{Particle identification and photo-detectors}

The ability to identify particles is imperative for the efficient tagging of quark flavours and for enhancing the signal from the background in selected final states. Prominent detector techniques, exploiting the detection of Cherenkov photons emitted by charged particles while traversing a radiator medium are the RICH and DIRC [42, 43]; they have succeeded in identifying particles across an impressively vast momentum range: from a few hundred $\mathrm{MeV} c$ up to several hundred $\mathrm{GeV} c$ [41]. HEP is populated by a number of Cherenkov detectors, some exhibiting extraordinarily complex optical paths, produced by exquisite manufacturing. Nevertheless, despite the fact that the radiator can be complicated, gaseous, solid, liquid, aerogel, warm or cold, it has never been a limiting factor for this technology: the conundrum always lies in the photo-detection plane. The present experiments are very demanding to the single-photon detectors; they require gain higher than $5 \times 10^{5}$, and sometimes this has to be achieved inside a high magnetic field, they necessitate time resolution much smaller than $100 \mathrm{ps}$ and a very high detection efficiency (owing to the limited number of emitted photons per traversing particle), they must exhibit long lifetime and rate stability in the range of several $\mathrm{MHz} \mathrm{cm}^{-2}$. Traditionally, experiments with small area single-photon detectors could afford solid-state detector planes, while large area experiments [44-46] always favoured gaseous detecting planes equipped with photo-cathodes; these detectors satisfy all the requirements imposed by the physics and are very advantageous in price, despite a certain complication in the manufacturing and the need for awareness during operation to master ageing effects. Recently, industry, has made available more and more sophisticated single-photon detectors, among others: the micro channel plate (MCP) with a collection efficiency of $50-55 \%$ and analogue or digital $\mathrm{SiPMs}^{3}$ that, if operated with light focalizers [47], can achieve a collection efficiency as high as $85 \%$. Figure 7 shows the single photon-electron peaks detected with a commercial digital SiPM, indicating the accuracy of the detector and the high reachable gain.

3 SiPM: a detector to be proud off! Yet another magnificent HEP in-house development $[48,49]$ that has now become an industrial standard with many applications outside our fundamental research world.

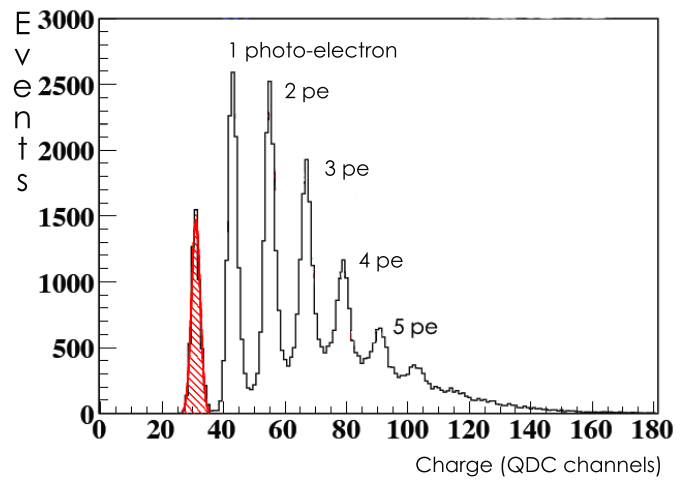

Figure 7. Single photon-electrons peaks detected with a commercial digital SiPM.

Although the price of commercial photo-detectors remains quite high, many experiments are actively considering their implementation since the monetary drawback is compensated by the convenient commercial availability of off-the-shelf products and the simplifications in the detector design. Still, certain features, like radiation hardness and temperature dependence, that are not very significant when using commercial photo-detectors outside HEP, but are fundamental in our experiments, have to be mastered, fostering yet more involvement and $R \& D$ with industry. Two very promising developments in the field are:

- the SiPM just produced in Chartered $130 \mathrm{~nm}$ Tezzaron $^{-1}$ 3D technologies which achieves a fill-factor of $92 \%$. A significant $R \& D$ effort is being invested to increase the fill-factor close to $100 \%$, and make these detectors an interesting solution for a pixel system at linear colliders [50];

- the large-area picosecond photo-detector (LAPPD) project (http://psec.uchicago.edu) [51], whose target is the development of large-area systems to measure the time-of-arrival of relativistic particles with (ultimately) $1 \mathrm{ps}$ resolution. The single detector unit is composed of a pair of novel, homemade, MCP manufactured by atomic layer deposition on a cheap substrate [52]. In this detector, the photoelectrons are accelerated across a proximity gap and then amplified in the MCPs; the resulting electron cloud is detected on anodic strips to 

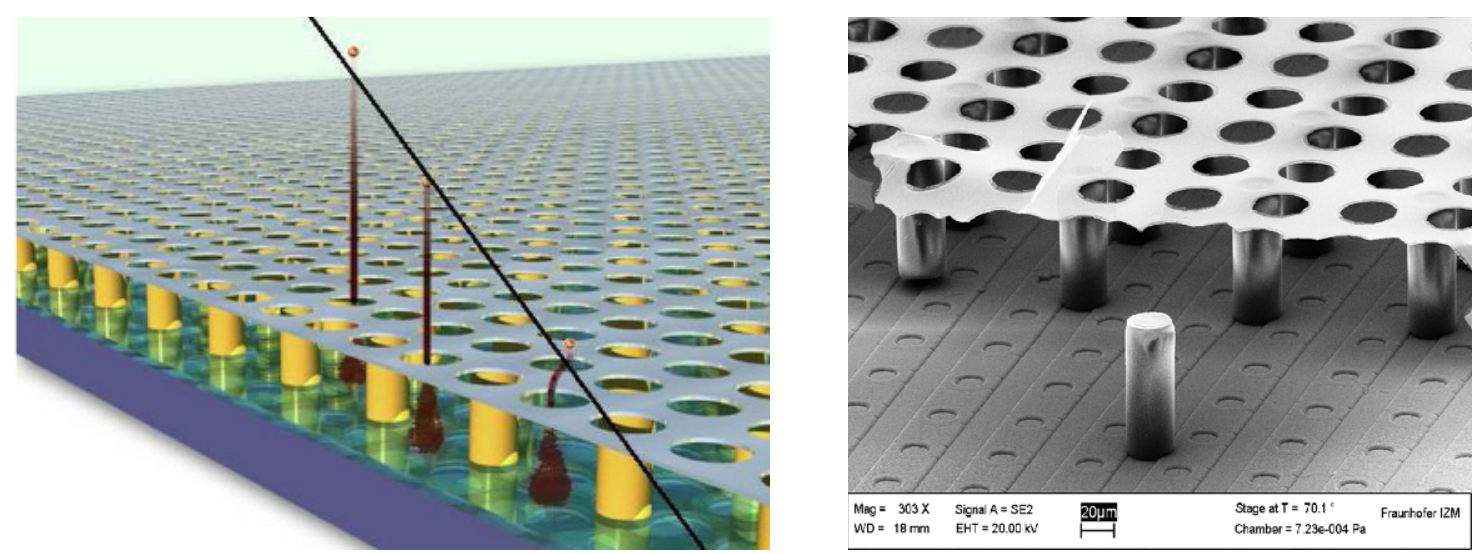

Figure 8. (Left) Schematic of a GridPix detector. Charges from an ionizing particle are drifted through a Micromega grid and amplified on top of a CMOS pixel chip. (Right) scanning electron microscope image of a real GridPix detector with the grid supported by the pillars (Courtesy of $\mathrm{H}$ van der Graaf).

determine the hit coordinates and the arrival time of the event. Recent tests demonstrate that the system is radiation tolerant up to at least $7 \mathrm{C} \mathrm{cm}^{-2}$ [53], i.e. twice the commercial MCP, reaches gains of $2 \times 10^{7}$, has a time-of-flight resolution of $35 \mathrm{ps}$ and a differential time resolution of $6 \mathrm{ps}$, corresponding to a spatial resolution of some $0.5 \mathrm{~mm}$ [54]. Each detector unit is $20 \times 20 \mathrm{~cm}^{2}$, i.e. very large compared with those available commercially; hence, the LAPPD project might offer very large area photo-detection planes at a reasonable price. If equipped with dedicated custom ASICs, LAPPD will have several very relevant potential applications: time-of-flight positron emission tomography, large water Cherenkov detectors, precision time-of-flight for a hadron collider, a preshower calorimeter and more.

\section{Gaseous detectors}

Progress in the microelectronics industry, photolithography and printed circuit technology paved the road to the production of MPGD that now take the baton from wire chambers. Nowadays there is quite a variety of MPGDs: strip-type, dot-type, hole-type and parallel-plate type [55]. MPGDs offer many advantages: unprecedented spatial resolution (down to tenths of a $\mu \mathrm{m}$ ), high-rate capability $\left(>10^{6} \mathrm{~Hz} \mathrm{~mm}^{-2}\right)$, large sensitive area, operational stability, increased radiation hardness, high time resolutions and good counting rate. GEM, MicroMega and some designs resulting from their evolution, have proven to be established technologies that meet the requirements for large area trackers [56, 57], TPC [58, 59], RICHs [60] and are under consideration as calorimeter active sampling planes for the experiments at the incoming linear collider. MPGDs are also widely considered for applications outside HEP: in astro-particle and neutrino physics, medical imaging, materials science and screening for homeland security. Among all of these many applications, two very aggressive ones are worth considering and following since they will certainly set a new standard in the gaseous detection realm:

- the future operation of the ALICE TPC, whit $\mathrm{Pb}-\mathrm{Pb}$ collision rates of $50 \mathrm{kHz}$. The existing MWPCs will be replaced by GEMs foils that operate with an intrinsic ion back flow suppression; they are just slightly less efficient than a classical ion-gating grid, that would introduce dead times and limit the trigger rates. Monte-Carlo simulations have demonstrated that the residual distortions from the space charge induced by the ion feedback are easily correctable by means of calibration techniques. Hence, it is expected that the new detection system will make it possible to increase the event rate by a factor of $100[61,62]$.

- the InGrid structure is an extraordinary single-electron sensitive monolithic device consisting of a MicroMegas directly integrated on top of a CMOS pixel chip by means of a wafer post-processing technology [63, 64]. By coupling this device to a thin gas drift gap (GridPix) and exploiting it with a high granularity (55 $\mu \mathrm{m}$ pitch) Medipix 2 or Timepix chip, the structure can be used as a very powerful TPC detector plane, as shown in figure 8 , or vertex detector that, compared to a conventional silicon pixel, would provide a low material budget and a low cooling power.

The extraordinary success and the many applications of MPGDs in diversified fields are certainly a remarkable achievement but, at the same time, they introduce a few worrisome key points that must be solved for further advances to be made: the cost-effective manufacturing of large-size MPGD detectors, the technology transfer and industrialization aspects of the production, and the capability of supplying a very large quantity of detectors in the upcoming years.

\section{Conclusions}

Detector R\&D is the vital backbone for the success of the upcoming large and complex HEP experiments. We might dispose, today, of several useful technologies, but certainly much progress has to be made to fulfil the demanding requirements of the upcoming machines. It is naive, and historically invalid, to believe that major breakthroughs would only happen from $R \& D$ performed within large collaborations where studies are, obviously, polarized by the internal challenges to be solved. Hence, foresightedly, it would be beneficial to invest manpower and fundings into a corporate and global organization devoted to the creation of new knowledge for the purpose of enabling new detectors, processes and services. 
Despite the diversity of the technologies that we handle, there are common needs and interest across several fields of particular relevance for detector research: the continuous push for higher granularity, radiation hardness, hermeticity, better timing resolution, higher trigger and data readout; and yet another reason to make all efforts to establish a global and coordinated programme that encompasses universities, laboratories and institutions.

The enormous progress in micro-processing and micro-electronic technologies of the past decade, has triggered a major transition in many detectors' components: from high quality 'in-house' manufacturing to industrial 'off-the-shelf' procurement. This trend, requires ample and coordinated R\&D since industrial products are, not always, qualified for application in our environment. Furthermore, when addressing the micro-electronic and especially the $3 \mathrm{D}-\mathrm{VI}$ or the deep-submicron technologies, that are both complex and involve high development costs, since our demand is extremely small compared to what is used by industry, their access might be prohibitive; hence, again, to cope with the cost, to share efforts and experience and to facilitate collaboration, the community needs to be organized and to advance within worldwide consortia.

\section{Acknowledgments}

The author thanks the organizers of 'The Nobel Symposium on LHC results' for the most challenging, interesting and exceedingly well organized conference. A special thank to the colleagues, and friends, for the constructive discussions and criticisms: Cinzia Da Via', Marcel Demarteau, Marcella Diemoz, Anna Macchiolo, Sandro Marchioro, Eugenio Nappi and Felix Sefkow.

\section{References}

[1] Charpak G et al 1968 Nucl. Instrum. Methods A 62 262-7

[2] Nygren D 1974 Proposal to investigate the feasibility of a novel concept in particle detection Lawrence Berkeley Laboratory Internal Report

[3] Nygren D and Marx J N 1978 Phys. Today 3146

[4] Seguinot J and Ypsilantis T 1977 Nucl. Instrum. Methods A $142377-15$

[5] Santonico R and Cardarelli R 1981 Nucl. Instrum. Methods A 377187

[6] Giomataris Y et al 1996 Nucl. Instrum. Methods A 37629

[7] Sauli F 1997 Nucl. Instrum. Methods A 386531

[8] Bouclier R et al 1997 IEEE Trans. Nucl. Sci. 44646

[9] Parker S, Kenney C and Segal J 1997 Nucl. Instrum. Methods A 395328

[10] ILD Concept Group 2010 ILD LoI DESY 2009-87 FNAL-PUB-09-682-E KEK Report 2009-6

[11] SiD Collaboration $2009 \mathrm{SiD}$ letter of intent arXiv:0911.0006v1 [physics.ins-det]

[12] Linssen L et al 2012 Physics and Detectors at CLIC: CLIC Conceptual Design Report ANL-HEP-TR-12-01, CERN-2012-003, DESY 12- 008, KEK Report 2011-7

[13] Buskulic D et al 1995 Nucl. Instrum. Methods A 360 481-506

[14] CMS Collaboration 2009 CMS-PAS-PFT-09-001

[15] Thomson M A 2009 Nucl. Instrum. Methods A 61125

[16] CALICE Collaboration 2007 Test Beams: The Data Taking, from 2007 Onwards (https://twiki.cern.ch/twiki/bin/ view/CALICE/TestBeamProgramme)

[17] Adloff C et al (CALICE Collaboration) 2012 J. Instrum. 7 P09017
[18] Da Via C and Battaglia M 2012 R\&D paths of pixel detectors for vertex tracking and radiation imaging Euro. Strategy Preparatory Group submitted

[19] Demarteau M et al 2009 Developments of novel vertically integrated pixel sensors in the high energy physics community Proc. IEEE Int. Conf. on 3D System Integration (December 2009) (San Francisco, CA: Freeman)

[20] Yarema $\mathrm{R}$ et al 2008 3D IC pixel electronics the next challenge TWEPP-08: Proc. of Topical Workshop on Electronics for Particle Physics (Naxos, Greece), 15-19 September 2008

[21] Moser H G 2012 3D Interconnection with TSV PoS (Vertex 2012) 024

[22] Barbero M et al 2012 J. Instrum. 7 P08008

[23] Klumpp A et al 2004 Japan. J. Appl. Phys. 43 7A 829-30

[24] Weigell P et al 2011 J. Istrum. 6 C12049

[25] Macchiolo A et al 2011 Nucl. Instrum. Methods A 650 145-49

[26] Snoeys W 2013 Nucl. Instrum. Methods A 731 125-30

[27] Baltay C et al 2009 Status of the chronopixel project arXiv:0902.2192 [physics.ins-det]

[28] Dannheim D 2012 Vertex-detector R\&D for CLIC PoS (Vertex 2012) 031

[29] Jones J et al 2005 A pixel detector for level-1 triggering at SLHC arXiv:physics/0510228 [physics.ins-det]

[30] Lipton R 2007 Nucl. Instrum. Methods A 579690

[31] Lipton R 2011 Nucl. Instrum. Methods A 636 160-3

[32] Pozzobon N 2013 Nucl. Instrum. Methods A 732 151-5

[33] Pesaresi M and Hall G J. Instrum. 5 C08003

[34] Macchiolo A et al 2013 Nucl. Instrum. Methods A (http://dx.doi.org/10.1016/j.nima.2013.04.077)

[35] Da Via C 2012 Nucl. Instrum. Methods A 694 321-30

[36] Dalla Betta G F 2012 3D silicon sensors: irradiation results PoS (Vertex 2012) 014

[37] Da Via C 2013 Nucl. Instrum. Methods A 699 18-21

[38] Casse G et al 2010 Nucl. Instrum. Methods A 624 401-4

[39] LaPedus M 2012 Intel: 100 billion required for IC manufacturing semiconductor Manufact. Desig. (http://semimd.com/)

[40] Marchioro S 2013 private communication

[41] Nappi E and Seguinot J 2005 Riv. Nuovo Cimento 28 8-9

[42] Ratcliff B 2003 Nucl. Instrum. Methods A 502 211-21

[43] Adam I et al 2005 Nucl. Instrum. Methods A 538281

[44] Arnold R et al 1988 Nucl. Instrum. Methods A 270255

[45] Gallas A et al 2007 Nucl. Instrum. Methods A 581402

[46] Albrecht E et al 2005 Nucl. Instrum. Methods A 553215

[47] Korpar S et al 2010 Nucl. Instrum. Methods A 613 195-4

[48] Bondarenko G et al 2000 Nucl. Instrum. Methods A 442187

[49] Dolgoshein B 2004 Proc. 42nd Workshop of the INFN ELOISATRON Project (Erice) (Singapore: World Scientific) p 442

[50] Vilella E et al 2013 Nucl. Instrum. Methods A 731 103-8

[51] Credo T et al 2004 Proc. IEEE Conf. on Nuclear Science Symp. vol 1

[52] Sullivan N et al 2009 Novel micro-channel plate device fabricated with atomic layer deposition Proc. 9th Int. Conf. on Atomic Layer Deposition (Monterey, 2009)

[53] Siegmund O H W et al 2013 IEEE Trans. Nucl. Sci. 602

[54] Adams B et al 2013 Nucl. Instrum. Methods A 732 392-6

[55] Sauli F 2002 Nucl. Instrum. Methods A 477 1-7

[56] ATLAS Collaboration 2011 Letter of intent for the phase-I upgrade of the ATLAS experiment CERN-LHCC-2011-012 LHCC-I-020

[57] Abbaneo D et al 2013 Nucl. Instrum. Methods A 718 383-3

[58] ALICE Collaboration 2013 Letter of intent for the upgrade of the ALICE experiment CERN-LHCC-2012-012; LHCC-I-022

[59] Attie D et al 2011 J. Instrum. 6, C01007

[60] Alexeev M et al 2013 J. Instrum. 8 P01021

[61] Kezner B et al 2013 Nucl. Instrum. Methods A 732 237-40

[62] Bohmer V F et al 2013 Nucl. Instrum. Methods A 719 101-7

[63] Campbell M 2005 Nucl. Instrum. Methods A 540 295-9

[64] Koppert W J C et al 2013 Nucl. Instrum. Methods A 732 245-9 